\begin{tabular}{|c|c|c|c|c|c|c|c|c|c|c|c|c|c|}
\hline \multirow{2}{*}{ Object } & \multirow{2}{*}{$\mathrm{Ph}$. } & \multirow{2}{*}{$\mathrm{Bb}$. } & \multicolumn{3}{|c|}{ M. Z. Strassburg } & \multirow{2}{*}{ Bem. } & \multirow{2}{*}{ Object } & \multirow{2}{*}{$\mathrm{Ph}$. } & \multirow{2}{*}{$\mathrm{Bb}$} & \multicolumn{3}{|c|}{ M. Z. Strassburg } & \multirow{2}{*}{ Bem. } \\
\hline & & & I. Berührung & Mitte & 2. Berühr. & & & & & I. Berührung & Mitte & 2. Berühr. & \\
\hline Archimedes & A. & $\mathrm{K}$ & $10^{\mathrm{h}} 5^{\mathrm{I}^{\mathrm{m}} 10^{\mathrm{s}}}$ & $5^{1^{m}} 49^{s}$ & $5^{2^{m}} 52^{s}$ & - & Posidonius & A. & $\mathrm{K}$ & $11^{h}-$ & - & $7^{\mathrm{m}} 40^{\mathrm{s}}$ & - \\
\hline Plato & $\Rightarrow$ & $\mathrm{K}$ & 105322 & $54 \quad 18$ & $545^{8}$ & - & Taruntius & $\gtrsim$ & $\mathrm{K}$ & $11-$ & - & 1045 & - \\
\hline & & $\mathrm{T}$ & - & - & 5424 & - & Proclus & 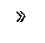 & $\mathrm{T}$ & $\mathbf{x} I-$ & $11^{\mathrm{m}} 32^{5}$ & - & - \\
\hline & & $\mathbf{E}$ & - & - & 5458 & - & & & $\mathbf{K}$ & - & I I 58 & - & - \\
\hline Manilius & $»$ & B & 10 - & 5521 & - & 14 & & & $\mathrm{~B}$ & - & 1230 & - & - \\
\hline Aristyllus & $\Delta$ & $\mathrm{K}$ & 10 - & - & $55 \quad 37$ & - & Picard & $\varnothing$ & $\mathrm{K}$ & $11-$ & I 4 I 8 & - & - \\
\hline Menelaus & $»$ & $\mathbf{K}$ & $10-$ & $5^{8} \quad 28$ & - & - & Condorcet & $\gg$ & $\mathrm{K}$ & I $1-$ & I 726 & - & - \\
\hline Plinius & 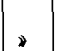 & $\begin{array}{l}\mathbf{I} \\
\mathrm{K}\end{array}$ & 115 & 23 & $\begin{array}{c}5844 \\
-\end{array}$ & - & Austritt des Rand & s & $\mathrm{E}$ & I I & 20 & - & 15 \\
\hline Dawes & » & $\mathrm{K}$ & I 1 & 342 & - & - & aus dem Kern & & $\mathrm{T}$ & - & 2114 & - & - \\
\hline Vitruvius & $»$ & $\mathbf{K}$ & I I - & 623 & - & - & schatten & & $\mathbf{K}$ & - & 2133 & - & - \\
\hline
\end{tabular}

Beobachter und Instrumente :

$\mathrm{B}=$ Becker, 6 2. Refractor, Vergr. 27.

$\mathrm{E}=$ Ebell, Cometensucher von Reinfelder und Hertel, Oeff. I 2 I mm, Vergr. 2 I.

$\mathrm{K}=$ Kobold, $18 \mathrm{z}$. Refractor, Vergr. 82 .

$\mathrm{T}=$ Tetens, Fernrohr von Merz, Oeffn. $100 \mathrm{~mm}$, Vergr. 70.

Bemerkungen.

Beob. B. Bei der verhältnissmässig und unerwartet grossen Schärfe, welche die Schattengrenze hatte und erst gegen das Ende der Finsterniss verlor, würde es sich empfohlen haben, an Stelle der Beobachtung der Ein- und Austritte der Flecken und Krater die Lage der Hörnerspitzen mikrometrisch zu messen; ich habe es unterlassen, weil die Zeit bereits sehr vorgerückt war, als der Mond aus den Wolken heryorkam. - I. Identificirung zweifelhaft. - 2. Zur Identificirung wurde nach Ende der Finsterniss das Faden. mikrometer angesetzt und beiläufig gemessen: Object - Tycho $p=327^{\circ} 9, \Delta=15^{\prime \prime}$. $-4 . a-b p=321^{\circ} \circ, \Delta=84^{\prime \prime}$. - 5. $b$-Tycho $p=275^{\circ} 1, A=882^{\prime \prime}$. -6 . $c$-Tycho $p=88.2, A={ }_{4} 4^{\prime \prime}$. $-7 \cdot d$-Tycho $p=84^{\circ} .2, A=$

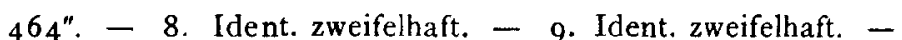
10. Ident. zweifelhaft. - I r. Obj. - Aristarch $p=26$ 198, $\Delta=225^{\prime \prime}$. - r 2. Obj. - Aristarch $p=257^{\circ} 2, A=349^{\prime \prime}$. - I3. Obj. - Aristarch $p=271 \%$ I, $\Delta=480^{\prime \prime}$. 14 . Ident. zweifelhaft.

Beob. E. 3. $\pm 20^{\mathrm{s}}$. - 15. Schon $2^{\mathrm{m}}$ vorher schien der Schatten den Mond zu verlassen.

Beob. K. Der Schattenrand war ziemlich deutlich und die Berührungen gut aufzufassen. Nur zum Schluss verlor der Schattenrand seine Schärfe und die letzten Austritte sind aus diesem Grunde ganz unsicher. Die angegebene Zeit des Endes kann um mehrere Minuten falsch sein.

\title{
Beobachtungen des Cometen 1898 I.
}

(Fortsetzung zu Nr. 3497).

\begin{tabular}{|c|c|c|c|c|c|c|c|c|c|c|c|}
\hline I 898 & M.Z.Hamb. & $\Delta \alpha$ & $\Delta \delta$ & Vgl. & Bb. & $\alpha$ app. & $\log p \cdot \Delta$ & $\delta$ app. & $\log p \cdot \Delta$ & Red. ad l. app. & $*$ \\
\hline April 27 & $12^{\mathrm{h}} 35^{\mathrm{m}} 35^{\mathrm{s}}$ & $+2^{m} 18.74$ & $+2{ }^{\prime} 17 \cdot 5$ & $23 \cdot 4$ & L & $0^{h} 15^{m} 16^{s} \cdot 35$ & $9.546_{\mathrm{n}}$ & $+48^{\circ} 58^{\prime} 53^{\prime \prime} 3$ & 0.884 & $+0.10-0.15$ & \\
\hline Mai 7 & 123542 & $+3 \quad 3.94$ & $\begin{array}{ll}-5 & 9.9\end{array}$ & 24.4 & S & 1947.25 & $9.548_{n}$ & +52569.0 & 0.882 & $+0.20+1.4$ & 2 \\
\hline I 1 & IO $40 \quad 21$ & -I 53.74 & -5 I 6.0 & 24.4 & $\mathbf{S}$ & $130 \quad 53.37$ & $8.867 \mathrm{n}$ & +535940.1 & 0.924 & $+0.28+2.2$ & 4 \\
\hline 21 & 10513 & +17.39 & $-6 \quad 37.2$ & 23.4 & S & $222 \quad 18.92$ & $8.865 n$ & $+55 \quad 4$ I 10.7 & 0.920 & $+0.55+3.0$ & 5 \\
\hline
\end{tabular}

Mittlere Oerter der Vergleichsterne.

\begin{tabular}{|c|c|c|c|c|c|c|c|}
\hline$*$ & $\alpha \mathrm{I} 898.0$ & $\delta 1898.0$ & Autorität & $*$ & $\alpha \quad 1898.0$ & $\delta_{1} 898.0$ & Autorität \\
\hline 1 & $0^{\mathrm{h}} 12^{\mathrm{m}} 57^{\mathrm{s}} \cdot 5 \mathrm{I}$ & $+48^{\circ} 5^{6^{\prime}} 36^{\prime \prime} 3$ & AG. Bonn 195 & 4 & $1^{h} 32^{m} 4^{6.8} 83$ & $+54^{\circ} 4^{\prime} 53^{\prime \prime} \cdot 9$ & AG. Cambridge $73^{8}$ \\
\hline 2 & I 643.11 & +53 I 17.5 & AG. Cambridge $55^{2}$ & 5 & $221 \quad 10.98$ & +554744.9 & AG. Hels. 2258 \\
\hline
\end{tabular}

Hamburg i 898 August. 